\title{
A squeeze method for hydrogen bond dynamics at instantaneous air-water interface
}

\author{
Gang Huang ${ }^{1}$ and Jie Huang ${ }^{2}$ \\ ${ }^{1}$ Institute für Physik, Johannes Gutenberg Universität \\ ${ }^{2}$ Department of Physics, Wenzhou University
}

May 19, 2021

\section{Hosted file}

main.pdf available at https://authorea.com/users/337426/articles/522578-a-squeeze-methodfor-hydrogen-bond-dynamics-at-instantaneous-air-water-interface 